\title{
Transcallosal, transchoroidal clipping of a hypothalamic collateral vessel aneurysm in Moyamoya disease
}

\author{
Wing Mann Ho ${ }^{1}$ - Alice Stephanie Görke ${ }^{1}$. Florian Dazinger ${ }^{2}$ • Bettina Pfausler ${ }^{3}$ Elke R. Gizewski ${ }^{2}$. Ondra Petr ${ }^{1}$. \\ Claudius Thomé ${ }^{1}$
}

Received: 3 March 2020 / Accepted: 7 April 2020 / Published online: 19 April 2020

(C) The Author(s) 2020

\begin{abstract}
Peripheral collateral vessel aneurysms in Moyamoya disease (MMD) remain difficult to treat due to their deep location, small size, and vascular fragility. We report the case of an aneurysm localized in the hypothalamus, which was rapidly increasing in size with repeated hemorrhage despite revascularization surgery. Aneurysm clipping was performed to prevent further progress and rerupture with favorable outcome. To our best knowledge, this is the first description of a hypothalamic aneurysm in MMD being clipped via a transcallosal, transchoroidal approach through the third ventricle.
\end{abstract}

Keywords Moyamoya $\cdot$ Aneurysm $\cdot$ Transcallosal approach $\cdot$ Clipping $\cdot$ Collateral vessel

\section{Introduction}

Moyamoya-like disease (MMD) is characterized by stenotic or hypoplastic internal carotid arteries (ICA) leading to altered flow dynamics and increased wall shear stress. This pathophysiologic mechanism is proposed to induce the formation of a fragile collateral network of Moyamoya vessels and associated aneurysms [18]. Those collateral aneurysms are usually small in size $(2-3 \mathrm{~mm})$ but have been associated with intraventricular hemorrhage (IVH) [2, 13-15]. Surgical revascularization has been observed with following obliteration of collateral artery aneurysms. The treatment of deep-seated

This article is part of the Topical Collection on Vascular Neurosurgery Arteriovenous malformation

Electronic supplementary material The online version of this article (https://doi.org/10.1007/s00701-020-04335-4) contains supplementary material, which is available to authorized users.

Ondra Petr

ondra.petr@yahoo.com

1 Department of Neurosurgery, Medical University Innsbruck, Anichstrasse 35, 6020 Innsbruck, Austria

2 Department of Neuroradiology, Medical University Innsbruck, Anichstrasse 35, 6020 Innsbruck, Austria

3 Department of Neurology, Medical University Innsbruck, Anichstrasse 35, 6020 Innsbruck, Austria peripheral artery aneurysms is technically highly challenging, with only few cases reported with favorable outcomes [9].

To our best knowledge, this is the first description of a collateral vessel aneurysm localized in the hypothalamus being clipped via a transcallosal, transchoroidal approach.

\section{Case report}

A 37 years-old female patient was admitted to the emergency room due to sudden severe headache with nausea, vomiting, and signs of meningeal irritation. She presented with anisocoria with a dilated right-sided pupil and a mild leftsided central facial palsy.

The initial computed tomography (CT) showed intracerebral hemorrhage (ICH) in the right hypothalamus with intraventricular hemorrhage (IVH) and subarachnoid hemorrhage (SAH) in the basal cisterns with a Hunt and Hess score of 3 and Fisher score of 4 (Fig. 1a). Additionally, CT angiography (CTA) was conducted arousing suspicion of MMD with a network of dense collateral arterial vessels (Fig. 1b).

The patient was admitted to the intensive care unit (ICU) and digital subtraction angiography (DSA) confirmed severe stenosis of both ICA and the A1 segments. Also, moderate stenosis of the left MCA was obvious with pronounced collaterals between the ICAs, both proximal ACAs and the right posterior communicating artery (PCOM). The dilated perforating vessels in the basal ganglia and hypothalamus were 

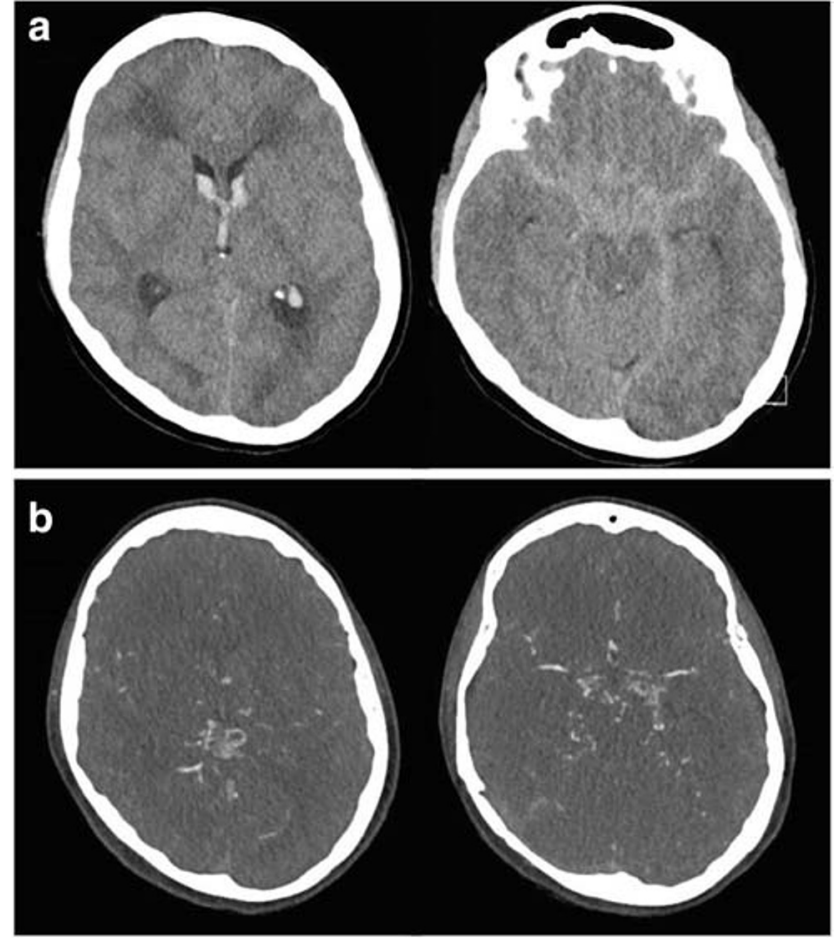

Fig. 1 a The initial CT scan demonstrating ICH in the right hypothalamus with IVH and SAH in the basal cisterns, scored as Hunt and Hess 3/Fisher 4. Additionally, signs of diffuse leukoencephalopathy in both hemispheres with spotty calcification were observed. b The CT angiogram demonstrated stenosis of both distal internal carotid arteries (ICA) and a proximally dilated posterior communicating artery, which appeared occluded after the P1 segment. The middle (MCA) and anterior cerebral arteries (ACA) were partially fed by collaterals with a rete of collateral moyamoya vessels

supposed to be caused by hypoperfusion due to MMD. One distinctive large collateral vessel was present between the reticular collaterals of the distal right-sided ICA and the ACA territory bearing a small aneurysm (Fig. 2). Given the primary

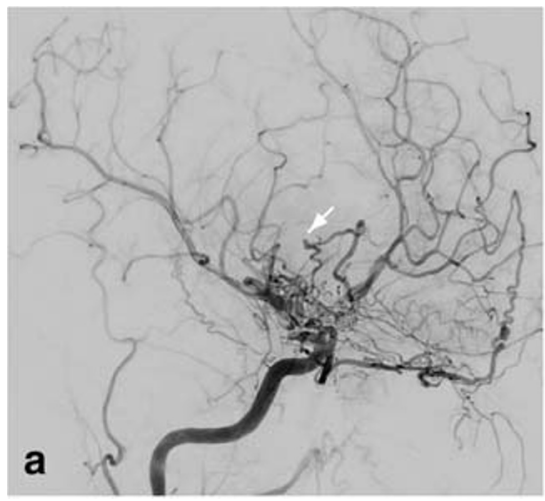

Fig. 2 The initial DSA confirming the network of moyamoya-pattern collateral vessels. The arrow points to the aneurysm and suspected bleeding source. Severe stenosis of both ICA and the A1 segments and moderate stenosis of the left MCA were obvious with pronounced collaterals between the ICAs, both proximal ACAs and the right posterior blood distribution, this $2 \mathrm{~mm}$-sized wide neck aneurysm was suspected as bleeding source. Thus, direct occlusion of the deep-seated aneurysm by means of either surgical or endovascular was considered to be associated with very high treatment-related morbidity, so that revascularization of the right hemisphere was indicated as the first step in order to reduce collateral perfusion in the perforating arteries. Due to a potentially increased perioperative risk in the acute phase after SAH and IVH, bypass surgery was scheduled 4 weeks later when the ICH had completely resorbed and the patient clinically recovered.

Blood pressure was monitored and systolic peak values above $140 \mathrm{mmHg}$ were prevented medically to avoid rebleeding. Nimodipine (dosage six times $60 \mathrm{mg}$ per day) was administered as a precaution against vasospasm/delayed cerebral ischemia and daily transcranial Doppler ultrasound revealed no signs of vasospasm. After 4 weeks, low-dose aspirin was started and standard extracranial-intracranial (ECIC) bypass surgery with a supplementary encephalomyosynangiosis was performed via a right-sided frontotemporal surgical approach. The patient rapidly recovered from surgery but complained about severe headache and nausea. Postoperative CT and CTA revealed a small rehemorrhage in the right hypothalamus again with intraventricular extension and a patent anastomosis. Additionally, a dissection of the left vertebral artery (VA) was discovered in the V3-segment and was treated with unfractionated heparin as standard of care. The underlying cause for the vessel dissection remained unclear. The following week, the patient was clinically stable without signs of vasospasm, ischemia, or hydrocephalus.

Postoperative DSA 1 week after surgery demonstrated proper bypass function with filling of the MCA territory and reduced perfusion of the network of collaterals around the ICA and proximal right-sided ACA. The aneurysm of the

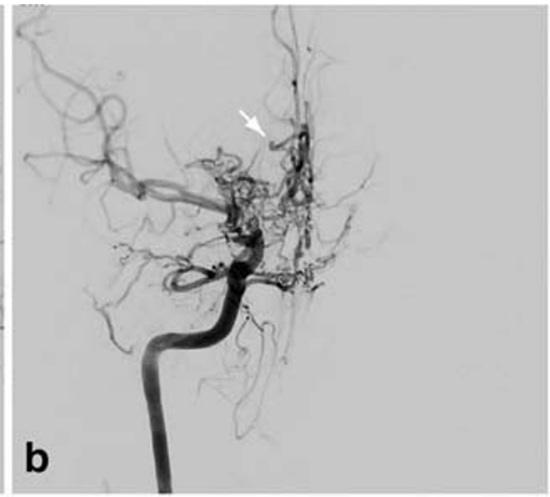

communicating artery (PCOM). The dilated perforating vessels in the basal ganglia and hypothalamus were supposed to be caused by hypoperfusion due to MMD. One distinctive large collateral vessel was present between the reticular collaterals of the distal right-sided ICA and the ACA territory bearing a small aneurysm 
Fig. 3 The 3D reconstruction of the follow-up CT angiogram showing the configuration of the collateral vessel aneurysm

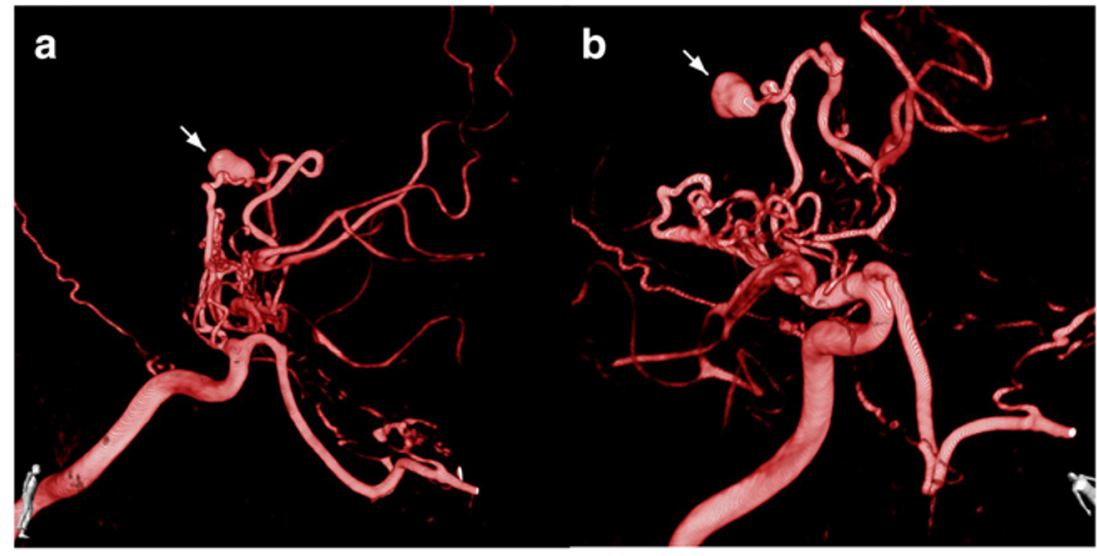

fragile collateral vessel, however, grew significantly within the following 3 weeks to a size of $7 \mathrm{~mm}$ (Fig. 3). In view of the rapid aneurysm growth and repeated hemorrhage, the rebleeding risk was assumed high and treatment was considered mandatory. Given the limited endovascular options with potential occlusion of the perforator and consecutive ACA ischemia, surgical exploration was indicated.

One month after EC-IC bypass surgery, the aneurysm was exposed using neuronavigation via an interhemispheric transcallosal, right-sided transchoroidal approach through the third ventricle. The aneurysm was surrounded by residual intraparenchymal hemorrhage and mobilized out of the hypothalamus, so that direct clipping could be achieved (Fig. 4). DSA confirmed complete aneurysm occlusion while showing the parent vessel intact (Fig. 5). The VA dissection was still stenotic but sufficiently collateralized via the contralateral side. There were no signs of hemorrhage or ischemia with no new symptoms or neurologic deficits postoperatively. The patient was discharged home neurologically intact and in a very good overall clinical condition.

\section{Discussion}

To the best of our knowledge, this is the first report of a direct clipping of a perforator aneurysm in the hypothalamus via an interhemispheric transcallosal approach. Of note, this approach has been described in the treatment of both arteriovenous and cavernous malformations $[3,5,6]$.

MMD-associated collateral artery aneurysms are usually treated indirectly by surgical revascularization causing aneurysm obliteration due to an alteration of flow-related vessel dynamics $[4,11,14,15,17,20]$. The timing of bypass surgery after the initial bleeding remains unclear. Kanamori et al. recommend the operation as soon as the patients are stable, since collateral artery aneurysms localized around the ventricular walls in MMD are prone to rerupture within 1 month [11]. In our case, we accordingly performed an EC-IC bypass, yet the aneurysm size significantly increased within a short period of time. Cases of aneurysm formation localized close to the anastomosis after bypass surgery have been documented [1, 7 , $8,16,19]$, but to date, only few cases of aneurysm formation and progression in collateral MMD vessels have been reported $[10,12,21]$.
Fig. 4 Intraoperative pictures via the third ventricular approach shows the aneurysm a in situ and b after microsurgical clipping

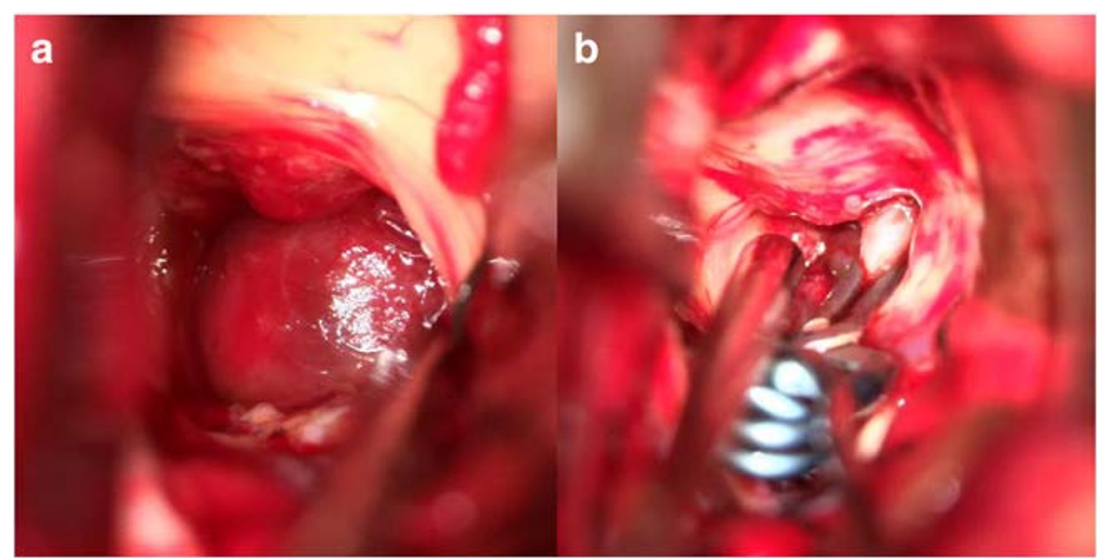



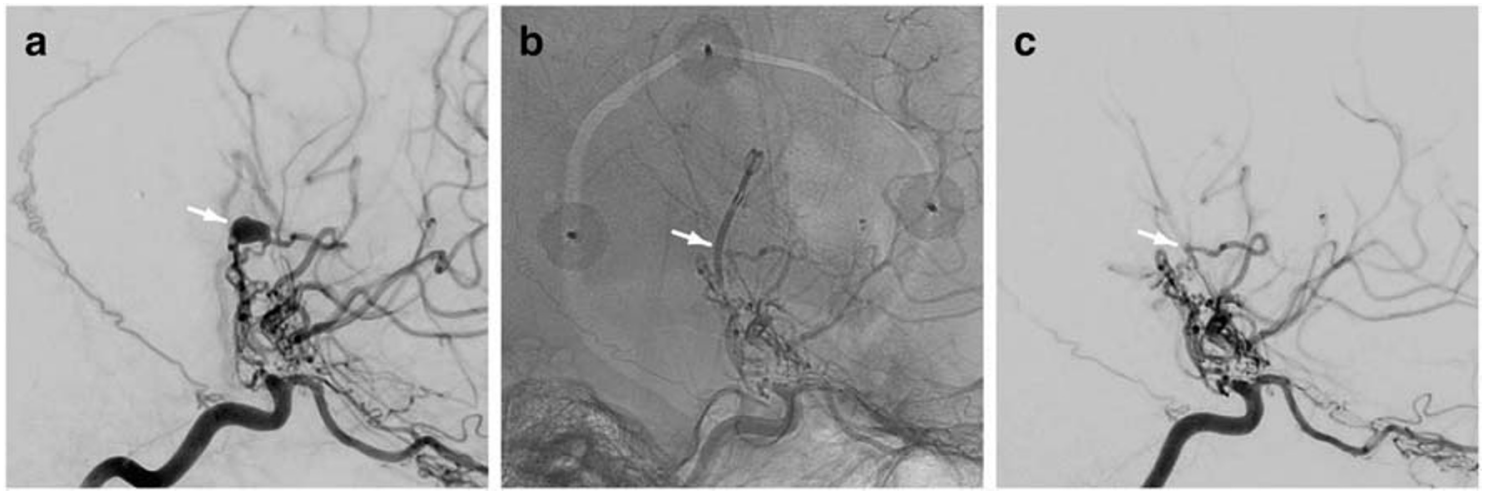

Fig. 5 The arrow in the DSA points to a the aneurysm before surgery, $\mathbf{b}$ the aneurysm clip, and $\mathbf{c}$ the complete obliteration of the aneurysm

The treatment of deep-seated peripheral artery aneurysms is technically challenging [22]. Either the feeding artery is hardly accessible for endovascular coiling due to the small size of the vessel, the fusiform character of the lesion and the fragile vascular structure, or the surgical approach may entail parenchymal damage of eloquent brain areas. Both treatment modalities bear significant risks of procedurerelated adverse events. Only few cases of deep-seated peripheral artery aneurysms have been reported with favorable outcomes [9]. In general, surgery within the hypothalamus is associated with a high risk of perioperative and postoperative morbidity including endocrinological and even neuropsychological deficits. In our case, the patient underwent direct aneurysm clipping without surgery-related complications and recovered completely without any neurological deficits. This may be due to the repeated intraparenchymal hemorrhage with rupture into the third ventricle, which has created an intraoperatively visible rather safe entry zone into the hypothalamus and may have eased the mobilization of the aneurysm into the third ventricle. Although proximal control could not be obtained without entering the hypothalamus further, intraoperative rupture was easily controlled, as flow in the perforators is somewhat limited. Therefore, surgical exploration is a feasible and safe option in cases in which fail revascularizations show repeated hemorrhage and/or are not amenable to endovascular treatment.

\section{Conclusion}

In cases of deep-seated peripheral collateral artery aneurysms localized in the hypothalamus, microsurgical clipping via a navigated interhemispheric transcallosal, transchoroidal approach through the third ventricle is technically feasible with favorable neurological outcome.

Funding Information Open Access funding provided University of Innsbruck and Medical University of Innsbruck.

\section{Compliance with ethical standards}

Conflict of interest The authors have no disclosures to declare. The patient's consent has been obtained for this publication.

Open Access This article is licensed under a Creative Commons Attribution 4.0 International License, which permits use, sharing, adaptation, distribution and reproduction in any medium or format, as long as you give appropriate credit to the original author(s) and the source, provide a link to the Creative Commons licence, and indicate if changes were made. The images or other third party material in this article are included in the article's Creative Commons licence, unless indicated otherwise in a credit line to the material. If material is not included in the article's Creative Commons licence and your intended use is not permitted by statutory regulation or exceeds the permitted use, you will need to obtain permission directly from the copyright holder. To view a copy of this licence, visit http://creativecommons.org/licenses/by/4.0/.

\section{References}

1. Aburakawa D, Fujimura M, Niizuma K, Sakata H, Endo H, Tominaga T (2017) Navigation-guided clipping of a de novo aneurysm associated with superficial temporal artery-middle cerebral artery bypass combined with indirect pial synangiosis in a patient with moyamoya disease. Neurosurg Rev 40(3):517-521

2. Adams HP, Kassell NF, Wisoff HS, Drake CG (1979) Intracranial saccular aneurysm and moyamoya disease. Stroke 10(2):174-179

3. Certo F, Toccaceli G, Altieri R, Barbagallo GMV (2019) Thalamomesencephalic cavernoma: anterior transcallosal transchoroidal approach. Neurosurgical focus: video 1(1):V20

4. Chen H, Hou K, Wang X, Xu K, Yu J (2019) Spontaneous recession of a posterior cerebral artery aneurysm concurrent with carotid rete mirabile and moyamoya-pattern collateral vessels: a case report. BMC Neurol. https://doi.org/10.1186/s12883-019-1277-7

5. Cossu G, González-López P, Daniel RT (2019) The transcallosal transchoroidal approach to the diencephalic-mesencephalic junction: how I do it. Acta Neurochir 161(11):2329-2334

6. Domingo RA, Grewal S, Tawk RG (2020) Interhemispheric Transcallosal approach for resection of choroidal arteriovenous malformation: operative video. World Neurosurg 136:73

7. Eom KS, Kim DW, Kang SD (2010) Intracerebral hemorrhage caused by rupture of a giant aneurysm complicating superficial temporal artery-middle cerebral artery anastomosis for moyamoya disease. Acta Neurochir 152(6):1069-1073

8. Fukushima Y, Miyawaki S, Inoue T, Shimizu S, Yoshikawa G, Imai H, Saito N, Tsutsumi K (2015) Repeated de novo aneurysm 
formation after anastomotic surgery: potential risk of genetic variant RNF213 c.14576G >A. Surg Neurol Int 6(1):41

9. Hamada J, Hashimoto N, Tsukahara T (1994) Moyamoya disease with repeated intraventricular hemorrhage due to aneurysm rupture. Report of two cases. J Neurosurg 80(2):328-331

10. Hayashi K, Horie N, Nagata I (2013) A case of unilateral moyamoya disease suffered from intracerebral hemorrhage due to the rupture of cerebral aneurysm, which appeared seven years later. Surg Neurol Int 4:17

11. Kanamori F, Takasu S, Ota S, Seki Y (2018) Prevention of the Rerupture of collateral artery aneurysms on the ventricular wall by early surgical revascularization in moyamoya disease: report of two cases and review of the literature. World Neurosurg 109:393397

12. Kim Y-S, Joo S-P, Lee G-J, Park J-Y, Kim S-D, Kim T-S (2017) Ruptured choroidal artery aneurysms in patients with moyamoya disease: two case series and review of the literatures. J Clin Neurosci 44:236-239

13. Kobayashi E, Saeki N, Oishi H, Hirai S, Yamaura A (2000) Longterm natural history of hemorrhagic type moyamoya disease in 42 patients. J Neurosurg 93(6):976-980

14. Kuroda S, Houkin K, Kamiyama H, Abe H (2001) Effects of surgical revascularization on peripheral artery aneurysms in moyamoya disease: report of three cases. Neurosurgery 49(2): 463-467 discussion 467-468

15. Ni W, Xu F, Xu B, Liao Y, Gu Y, Song D (2012) Disappearance of aneurysms associated with moyamoya disease after STA-MCA anastomosis with encephaloduro myosynangiosis. J Clin Neurosci 19(3):485-487
16. Nishimoto T, Yuki K, Sasaki T, Murakami T, Kodama Y, Kurisu K (2005) A ruptured middle cerebral artery aneurysm originating from the site of anastomosis 20 years after extracranialintracranial bypass for moyamoya disease: case report. Surg Neurol 64(3):261-265

17. Peltier J, Vinchon M, Soto-ares G, Dhellemmes P (2008) Disappearance of a middle cerebral artery aneurysm associated with Moyamoya syndrome after revascularization in a child: case report. Childs Nerv Syst 24(12):1483-1487

18. Scott RM, Smith ER (2009) Moyamoya disease and moyamoya syndrome. N Engl J Med 360(12):1226-1237

19. Tang C-L, Shen C-C (2016) Anastomotic aneurysm formation after superficial temporal artery - middle cerebral artery bypass surgery in 5 months. Formosan J Surg 49(4):149-153

20. Yamada H, Saga I, Kojima A, Horiguchi T (2019) Short-term spontaneous resolution of ruptured peripheral aneurysm in moyamoya disease. World Neurosurg 126:247-251

21. Yokota H, Yokoyama K, Noguchi H (2016) De novo aneurysm associated with superficial temporal artery to middle cerebral artery bypass: report of two cases and review of literature. World Neurosurg 92:583.e7-583.e12

22. Zhang L, Xu K, Zhang Y, Wang X, Yu J (2015) Treatment strategies for aneurysms associated with moyamoya disease. Int J Med Sci 12(3):234-242

Publisher's note Springer Nature remains neutral with regard to jurisdictional claims in published maps and institutional affiliations. 